\title{
Cost-Effectiveness of Dual Bronchodilator Indacaterol/Glycopyrronium for COPD Treatment in China
}

This article was published in the following Dove Press journal: International Journal of Chronic Obstructive Pulmonary Disease

\author{
Shiyi Gong' \\ $\mathrm{HaO} \mathrm{Hu}^{2}$ \\ Kun Zhao ${ }^{2}$ \\ Ting Yang iD ' \\ 'Department of Pulmonary and Critical \\ Care Medicine, China-Japan Friendship \\ Hospital; National Clinical Research \\ Center for Respiratory Disease; Institute \\ of Respiratory Medicine, Chinese \\ Academy of Medical Science; Peking \\ University Health Science Center, Beijing, \\ People's Republic of China; ${ }^{2}$ China \\ National Health Development Research \\ Center, National Health Commission of \\ the People's Republic of China, Beijing, \\ People's Republic of China
}

Background: Indacaterol/glycopyrronium (IND/GLY) is a once-daily dual bronchodilator for long-term treatment of patients with chronic obstructive pulmonary disease (COPD). The efficacy and safety of IND/GLY have been proved before, but the cost-effectiveness is unknown in China. Purpose: This study assessed cost-effectiveness of IND/GLY comparing with salmeterol/ fluticasone (SAL/FLU) and tiotropium.

Methods: A patient-level simulation model was established from Chinese payer perspective. Patient parameters were randomly simulated through resampling from parameter distributions based on clinical trials and China-specific cost data to represent individual level health state and health state transitions in the model. We simulated patient-level health state, costs, life years (LYs) and quality-adjusted life years (QALYs) of whole life horizon to evaluate the cost-effectiveness of IND/GLY comparing with SAL/FLU and tiotropium respectively.

Results: Comparing with SAL/FLU, IND/GLY resulted in 0.384 LYs and 0.255 QALYs gained. The incremental cost-effectiveness ratio (ICER) is $-35,822 \mathrm{CNY} / \mathrm{LY}$ and the incremental costutility ratio (ICUR) is $-53,834 \mathrm{CNY} / \mathrm{QALY}$ for IND/GLY versus SAL/FLU. Comparing with tiotropium, IND/GLY resulted in 0.232 LYs and 0.146 QALYs gained. The ICER is 39,729 CNY/ LY and the ICUR is 63,246 CNY/QALY for IND/GLY versus tiotropium.

Conclusion: This study found that dual bronchodilator IND/GLY is cost-effective for stable COPD treatment in China from Chinese payer's perspective.

Keywords: chronic obstructive pulmonary disease; COPD, dual bronchodilator, costeffectiveness analysis, China

\section{Introduction}

Chronic obstructive pulmonary disease (COPD) is one of most common chronic diseases in China. ${ }^{1-3}$ There are nearly 100 million COPD patients in China. ${ }^{1}$ COPD ranked in the top three leading cause of death in China. ${ }^{2,4}$ Each COPD patient in China spends 499 to 1932 USD on total costs annually. ${ }^{2}$ Besides medical costs increasing, COPD patients have worse health status and worse quality of life compared with the normal population. ${ }^{5}$ Thehuge disease burden of COPD calls for more attention to long-term management of COPD patients in China.

In China, current inhaled treatments for stable COPD include bronchodilators and inhaled corticosteroids (ICS). ${ }^{6}$ Monotherapies of bronchodilators, including long-acting beta2/adrenergic receptor agonists (LABAs) and long-acting cholinergic/muscarinic antagonists (LAMAs), are used for initial treatment in mild to moderate patients, ${ }^{6}$ while a combination of ICSs and LABA (ICS/LABA) is recommended for COPD patients with
Correspondence: Ting Yang Department of Pulmonary and Critical Care Medicine, China-Japan Friendship Center for Respiratory Disease; Peking Sealth Science Center; of Respiratory Medicine, Chinese Academy of Medical Science, Peking University Health Science Center, No. 2 Yinghua East Street, Chaoyang District, Beijing, People's Republic of China

Tel +86 I365I380809

Email zryyyangting@I63.com

International Journal of Chronic Obstructive Pulmonary Disease 2021:I6 433-44| 
high exacerbation risk or high blood eosinophil count. ${ }^{6}$ These inhaler therapies significantly improve lung function, reduce exacerbations and improve quality of life for COPD patients. In addition, combination therapy of two types of long-term bronchodilators (LAMA and LABA) performed better recently in clinical trials, ${ }^{7-11}$ hence it becomes a new choice for stable COPD patients with more severe symptoms. ${ }^{12}$ IND/ GLY, a once-daily dual bronchodilator for treatment of patients with COPD, which contains a fixed dose of 110ug of LABA indacaterol and $50 \mathrm{ug}$ the LAMA glycopyrronium. Compared with monotherapy bronchodilators and ICS/LABA therapy, IND/GLY significantly improves lung function, reduces exacerbation and improves health status in trials in China and worldwide. ${ }^{13-16}$ Previous studies have shown costeffectiveness of IND/GLY compared with other therapies from the payer's perspective in USA and Europe. ${ }^{17,18}$ However, China's medical system and medical service prices are completely different from the Western world. ${ }^{19}$ The costeffectiveness of IND/GLY should be reassessed in the setting of China's medical system.

In this study, we aimed to assess the cost-effectiveness of a LABA/LAMA combination therapy IND/GLY for COPD management compared with the current single LAMA therapy and ICS/LABA combination therapy from Chinese payer's perspective using real-world data in China.

\section{Method}

\section{Patient-Level Simulation Model}

A patient-level simulation is a type of model that simulated health state transition at patient-level rather than cohort-level. In this model, patients entering the model were generated randomly with specific parameters of demographic data and health outcomes to represent different health state. Patientlevel outcomes were determined based on random selection from specific distributions of parameters for target population with the model running. When compared with the traditional cohort-level Markov model, patient-level simulation model is assumed to be more flexible and more intuitive, as this model can capture heterogeneity in the patient population and simulate more health states. ${ }^{23}$

\section{Model Structure}

We developed a patient-level simulation model for COPD patients using Microsoft Excel ${ }^{\circledR}$ software to simulate health states, costs and outcomes at individual level over time as shown in Figure 1. One thousand individual-level virtual "patients" were randomly generated in this model.
Parameters that represent individual level health state were randomly assigned from the distributions derived from clinical trials and China-specific cost data. In the simulation, generated patients experienced disease progression and clinical events in every 6-month cycle in this model. Total costs, life years (LYs) and quality-adjusted life years (QALYs) of each patient was cumulated during time horizon of whole life time to evaluate the cost-effectiveness of IND/GLY and comparators respectively.

\section{Model Parameters}

IND/GLY as a representative of LABA/LAMA combination inhalation therapy, were compared with two another two current inhaled drugs for COPD. ICS/LABA combination salmeterol/fluticasone (SAL/FLU) $(50 / 500 \mu \mathrm{g}$, twice daily) and LAMA tiotropium $(18 \mu \mathrm{g}$, once daily) are most common inhaled drugs that prescribed by Chinese physicians. The baseline characteristics and clinical parameters of were derived from LANTERN trial for the comparison of IND/GLY and SAL/FLU. ${ }^{14}$ The baseline characteristics and clinical parameters were derived from SPARK trial for the comparison of IND/GLY and tiotropium. ${ }^{13}$ The baseline characteristics of cohort patients in this model are listed in Table 1. Clinical model input parameters of different treatment pattern in this model are listed in Table 2.

\section{Lung Function}

In the model, lung function improvement is captured as an increase in the actual FEV1 in liters (L) from the clinical trial and converted to a treatment effect versus placebo. We assumed that the lung function benefit occurs at the first 6-month cycle in this model. The FEV1 in liters declined over times and GOLD severity stage of patients updated every cycle in the model. The annual rate of lung function (FEV1) of patients was determined by their age and height by an equation (Supplementary Materials, Equation 1).2, ${ }^{20}$ Two FEV1 in liters of each patient were updated every cycle as the model was running.

\section{Clinical Events}

Different therapies have different effect of avoiding exacerbations and pneumonias. Acute exacerbation of COPD (AECOPD) is defined as acute episodes of breathlessness and sputum production that requiring additional treatment. We used exacerbation and pneumonia ratios, which is exacerbation and pneumonia rates of divided by exacerbation and pneumonia rates of placebo to represent treatment effect of adverse event-avoiding. Background 


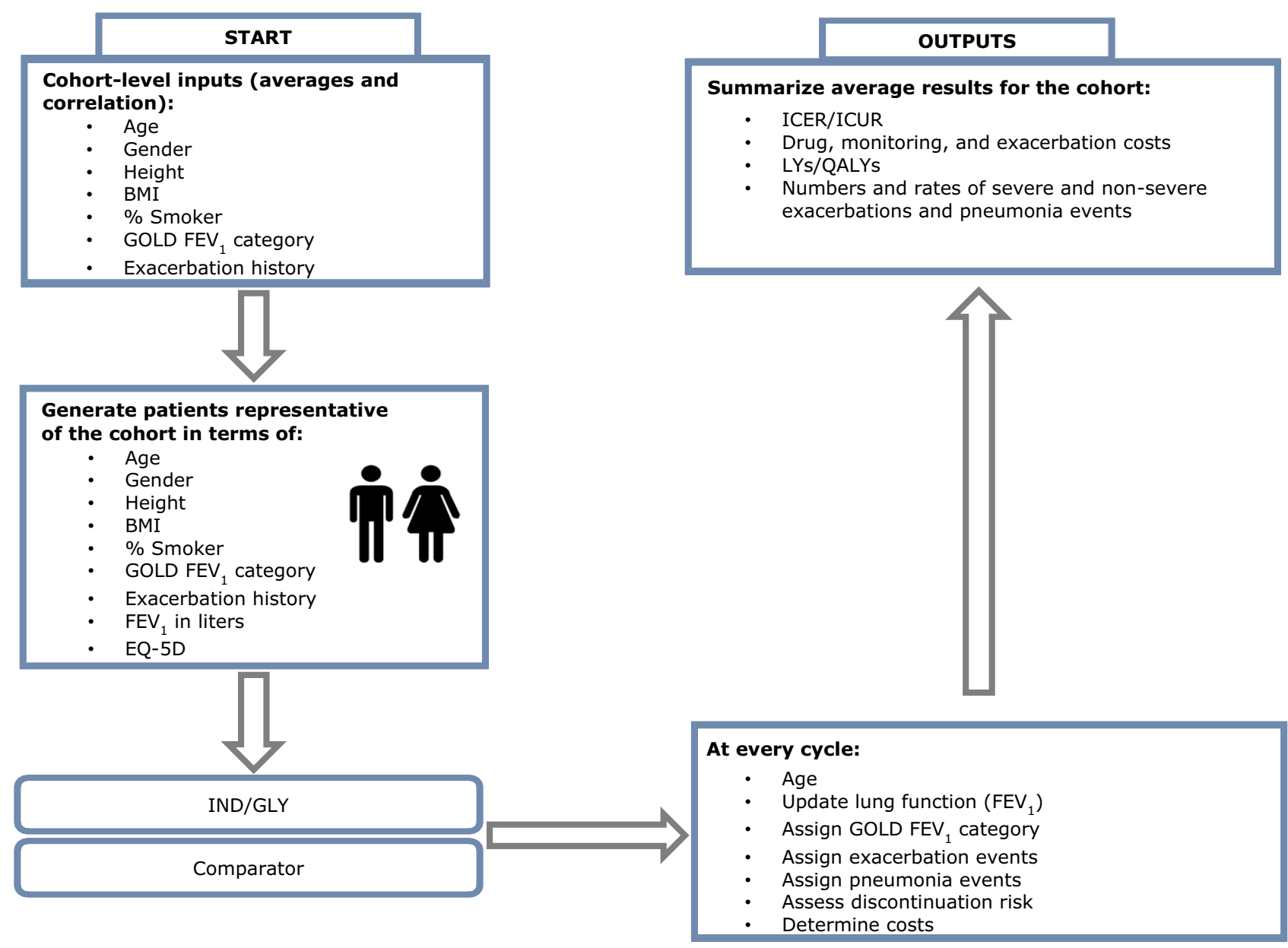

Figure I Model structure.

Abbreviations: BMI, body mass index; EQ-5D, EuroQol-Five Dimension; IND/GLY, indacaterol/glycopyrronium; ICER, incremental cost-effectiveness ratio; ICUR, incremental cost-utility ratio; LYs, life years; QALYs, quality-adjusted life years.

exacerbation or pneumonia rates for placebo multiplied by exacerbation ratios of specific treatment pattern come to actual exacerbation or pneumonia rates. Death events occur based on the lifetable of all-cause mortality for normal population in China. A hazard ratio was then applied to this mortality rate as a function of declining FEV1. For every $10 \%$ decline in FEV1\% predicted, there is a $2 \%$ increase in mortality risk. Clinical adverse event of acute exacerbation of COPD (AECOPD), pneumonia and death may occur randomly based on the distribution of the ratio of each event at every cycle as model running.

\section{Cost}

Drug costs of IND/GLY and comparators were sourced from Health Information System in a tertiary hospital in Beijing, China. Stable period costs and clinical event associated costs were derived from a survey of clinicians from 30 hospitals across the country. Based on their clinical experience, physicians estimated the costs of stable treatment, acute exacerbation and pneumonia for patients of different levels. In this model, we use the median of physicians' estimated value as parameters.

\section{Model Outcomes}

LYs and QALYs were the primary outcomes of different therapy patterns in this model. QALYs is the sum of LYs weighed by health utility of the health state of each LY. Health utility of COPD patients in different health states was calculated based on a published equations (Supplementary Materials, Equation 2). ${ }^{21}$ The cost effectiveness of IND/GLY versus comparators was determined by calculating incremental total cost per unit effectiveness gained of IND/GLY. We called the ratio of incremental costs and incremental QALYs as incremental cost-utility ratio (ICUR) when we used QALYs as effectiveness. According to standard of $\mathrm{WHO}$, an intervention with 
Table I Baseline Characteristics of Two Trial Cohort

\begin{tabular}{|l|l|l|}
\hline & $\begin{array}{l}\text { LANTERN }^{14} \text { (IND/ } \\
\text { GLY versus SAL/FLU) }\end{array}$ & $\begin{array}{l}\text { SPARK } \\
\text { versus Tiotropium) }\end{array}$ \\
\hline Age (years) & 65.09 & 63.33 \\
Gender (\% male) & $91 \%$ & $75 \%$ \\
Height (cm) & 165.69 & 168.75 \\
BMI (kg/m $\left.{ }^{2}\right)$ & 23.76 & 25.29 \\
Current smokers (\%) & $26 \%$ & $37 \%$ \\
Exacerbations in & 0.21 & 1.31 \\
previous year & \multicolumn{2}{|l}{} \\
\hline GOLD severity & \multicolumn{2}{|l|}{} \\
\hline GOLD I stage & $0 \%$ & $0 \%$ \\
GOLD II stage & $53 \%$ & $0 \%$ \\
GOLD III stage & $47 \%$ & $79 \%$ \\
GOLD IV stage & $0 \%$ & $21 \%$ \\
\hline
\end{tabular}

Abbreviations: IND/GLY, indacaterol/glycopyrronium; SAL/FLU, salmeterol/fluticasone; BMI, body mass index; GOLD, Global Initiative for Chronic Obstructive Lung Disease.
ICUR more than 1-3 times GDP per capita was assumed as cost-effective. ${ }^{22}$ This threshold to judge costeffectiveness is so-called willingness-to-pay (WTP). According to the data from the World Bank, the GDP per capita of China in 2019 was 10,261.68 USD, which was equal to $68,617.80 \mathrm{CNY}$. We selected a lower WTP threshold for chronic diseases like COPD. We used 70,000 CNY as the WTP threshold in this model to assess costeffectiveness, which is approximate to one-time GDP per capita. All costs and outcomes were discounted as a rate of $3 \%$ annually.

\section{Uncertainty Analysis}

Both one-way sensitivity analyses (OWSA) and probabilistic sensitivity analyses (PSA) were conducted to evaluate uncertainty of the model results. For OWSA, we adjusted

Table 2 FEVI in Liters Improvement in Two Cohorts

\begin{tabular}{|c|c|c|c|c|}
\hline Items & Mean & Lower $\mathbf{C l}$ & Upper Cl & Source \\
\hline FEVI improvement of LANTERN trial ${ }^{14}(\mathrm{~L})$ & $\begin{array}{l}\text { IND/GLY: } 0.210 \\
\text { SAL/FLU: } 0.103\end{array}$ & $\begin{array}{l}0.121 \\
-0.012\end{array}$ & $\begin{array}{l}0.299 \\
0.218\end{array}$ & 14 \\
\hline FEVI improvement of SPARK trial ${ }^{13}(\mathrm{~L})$ & $\begin{array}{l}\text { IND/GLY: } 0.190 \\
\text { Tiotropium: } 0.130\end{array}$ & $\begin{array}{l}0.110 \\
0.050\end{array}$ & $\begin{array}{l}0.270 \\
0.210\end{array}$ & 13 \\
\hline Background exacerbation rates ${ }^{15}$ & $\begin{array}{l}\text { Non-severe:1.010 } \\
\text { Severe: } 1.010\end{array}$ & $\begin{array}{l}0.920 \\
0.920\end{array}$ & $\begin{array}{l}1.340 \\
1.340\end{array}$ & 15 \\
\hline Exacerbation ratios versus placebo (LANTERN trial) $)^{14}$ & $\begin{array}{l}\text { IND/GLY:0.450 } \\
\text { SAL/FLU:0.925 }\end{array}$ & $\begin{array}{l}0.410 \\
0.500\end{array}$ & $\begin{array}{l}0.490 \\
1.350\end{array}$ & 14 \\
\hline Exacerbation ratios versus placebo $\left(\right.$ SPARK trial) ${ }^{13}$ & $\begin{array}{l}\text { IND/GLY:0.820 } \\
\text { Tiotropium: } 0.910\end{array}$ & $\begin{array}{l}0.660 \\
0.730\end{array}$ & $\begin{array}{l}0.980 \\
1.090\end{array}$ & 13 \\
\hline Background pneumonia rates & $\begin{array}{l}\text { Non-severe:0.050 } \\
\text { Severe:0.050 }\end{array}$ & $\begin{array}{l}0.050 \\
0.050\end{array}$ & $\begin{array}{l}0.050 \\
0.050\end{array}$ & 27 \\
\hline Pneumonia ratios versus placebo & $\begin{array}{l}\text { IND/GLY: } 1.000 \\
\text { SAL/FLU: } 3.430 \\
\text { Tiotropium: } 1.000\end{array}$ & $\begin{array}{l}0.900 \\
0.940 \\
1.000\end{array}$ & $\begin{array}{l}1.100 \\
12.460 \\
1.000\end{array}$ & $13,14,27$ \\
\hline Drug cost per day (CNY) & $\begin{array}{l}\text { IND/GLY: } 8.77 \\
\text { SAL/FLU: } 10.00 \\
\text { Tiotropium: } 6.00\end{array}$ & & & Survey \\
\hline Annual stable period cost (CNY) & $\begin{array}{l}\text { GOLD I: } 1000 \\
\text { GOLD II: } 2000 \\
\text { GOLD III: } 3000 \\
\text { GOLD IV: } 4000\end{array}$ & & & Survey \\
\hline Exacerbation cost (CNY) & $\begin{array}{l}\text { Non-severe: } 5000 \\
\text { Severe: } 8000\end{array}$ & & & Survey \\
\hline Pneumonia cost (CNY) & 6000 & & & Survey \\
\hline
\end{tabular}

Abbreviations: IND/GLY, indacaterol/glycopyrronium; SAL/FLU, salmeterol/fluticasone; FEVI, forced expiratory volume in I second; GOLD, Global Initiative for Chronic Obstructive Lung Disease; CNY, China Yuan. 
several individual parameters by $\pm 25 \%$ and reassessed the costs and effectiveness of IND/GLY and its comparators while maintaining all other parameters in this model. For the PSA, in which all parameters change spontaneously based on distributions of parameters after 100 iterations. The probability of acceptance of cost-effectiveness of IND/ GLY at the willing-to-pay of $70,000 \mathrm{CNY}$ was assessed.

\section{Result}

\section{IND/GLY versus SAL/FLU}

Compared with SAL/FLU, IND/GLY resulted in 0.384 LYs and 0.255 QALYs gained during lifetime simulation. Besides, total cost of IND/GLY was 13,754 CNY lower than SAL/FLU. The incremental cost-effectiveness ratio (ICER) is $-35,822 \mathrm{CNY} / \mathrm{LY}$ and the ICUR is $-53,834$ CNY/QALY for IND/GLY versus SAL/FLU. IND/GLY is considered more cost-effective (Table 3).

OWSA showed that FEV1 benefit, exacerbation rate ratio and drug cost sensitively influenced the results in this model. While the results were less sensitive in baseline exacerbation rates. (Supplementary Materials, Table S1) PSA showed that IND/GLY is dominantly cost-effective than SAL/FLU (Figure 2).
Table 3 Results of Whole Life Simulation of IND/GLY vs SAL/ FLU

\begin{tabular}{|l|l|l|l|}
\hline & IND/GLY & SAL/FLU & Incremental \\
\hline LYs & 10.772 & 10.388 & 0.384 \\
QALYs & 6.762 & 6.506 & 0.255 \\
All exacerbations & 4.390 & 6.060 & -1.670 \\
Total costs & 77,387 & 91,140 & $-13,754$ \\
Costs/LYs & & & $-35,822$ \\
ICUR & & & $-53,834$ \\
\hline
\end{tabular}

Abbreviations: IND/GLY, indacaterol/glycopyrronium; SAL/FLU, salmeterol/fluticasone; QALYs, quality-adjusted life years; LYs, life years; ICUR, incremental costutility ratio.

\section{IND/GLY versus Tiotropium}

Comparing with Tiotropium, IND/GLY resulted in 0.232 LYs and 0.146 QALYs gained during lifetime simulation. The total cost of IND/GLY was $9203 \mathrm{CNY}$ higher than Tiotropium. The ICER is $39,729 \mathrm{CNY} / \mathrm{LY}$ and the ICUR is 63,246 CNY/QALY for IND/GLY versus tiotropium. IND/ GLY is considered more cost-effective (Table 4).

OWSA showed that FEV1 benefit, exacerbation rate ratio and drug cost sensitively influenced the results in this model. While the results were less sensitive in baseline exacerbation rates. (Supplementary Materials, Table S2)

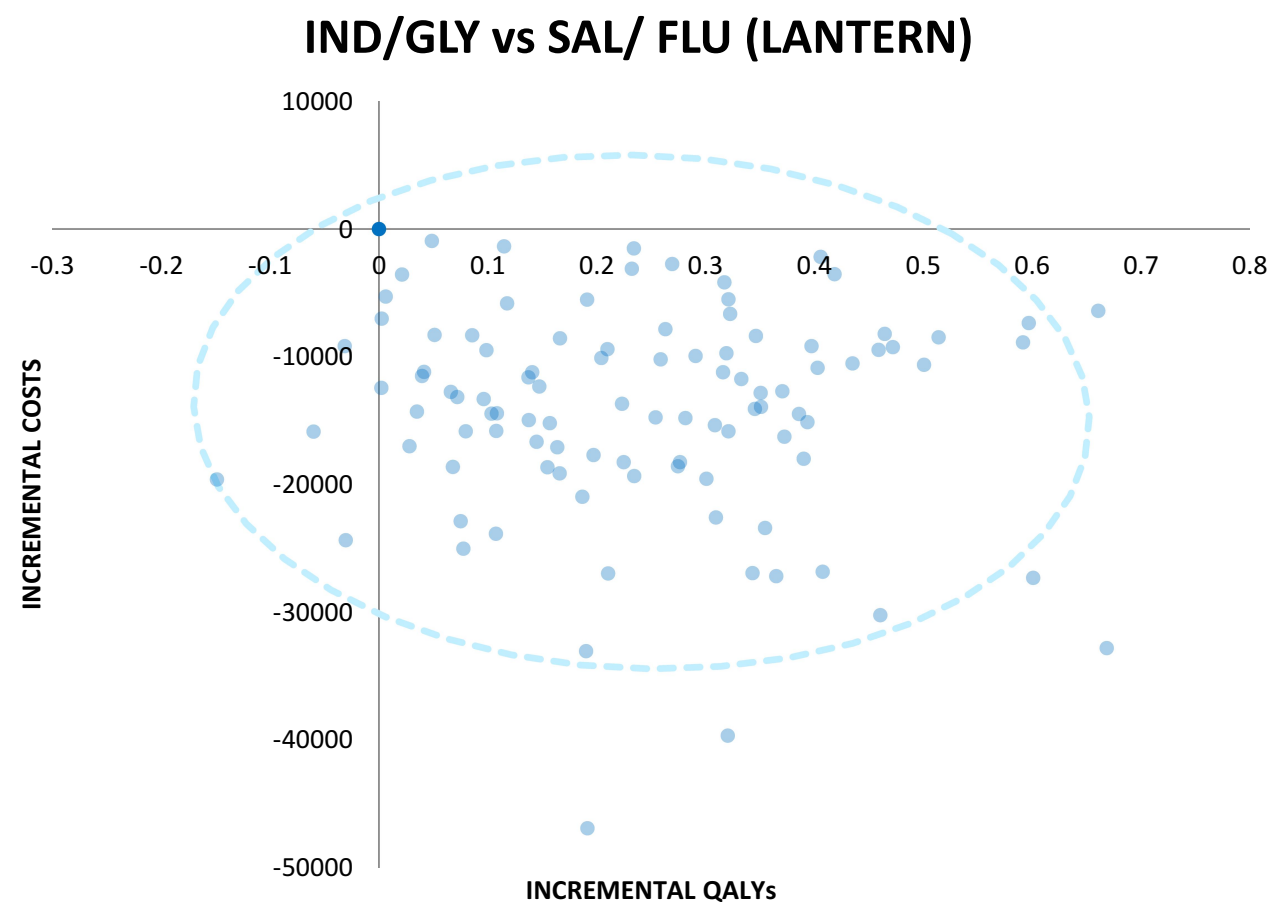

Figure 2 Probability sensitivity analysis of IND/GLY vs SAL/FLU. We showed the results of probability sensitivity analysis (PSA) of IND/GLY vs SAL/FLU in this scatter plot. Each scatter represents an iteration in this model with different incremental costs and QALYs in PSA. This figure showed in most iterations, IND/GLY is with lower costs and longer QALYs than SAL/FLU. We concluded that IND/GLY is with dominate cost-effectiveness than SAL/FLU. 
Table 4 Results of Whole Life Simulation of IND/GLY vs Tiotropium

\begin{tabular}{|l|l|l|l|}
\hline & IND/GLY & Tiotropium & Incremental \\
\hline LYs & 10.712 & 10.480 & 0.232 \\
QALYs & 6.702 & 6.556 & 0.146 \\
All exacerbations & 6.400 & 6.760 & -0.360 \\
Total costs & 85,730 & 76,528 & 11,346 \\
Costs/LYs & & & 39,729 \\
ICUR & & & 63,246 \\
\hline
\end{tabular}

Abbreviations: IND/GLY, indacaterol/glycopyrronium; SAL/FLU, salmeterol/fluticasone; QALYs, quality-adjusted life years; LYs, life years; ICUR, incremental costutility ratio.

PSA showed that IND/GLY has $50-60 \%$ chance of being cost-effective at the WTP of 70,000 CNY (Figures 3 and 4).

\section{Discussion}

In this study, we found that IND/GLY had better clinical effectiveness and lower costs than SAL/FLU. When compared with tiotropium, IND/GLY had better effectiveness and higher costs compared with TIO. Results showed that IND/GLY is moderately cost-effective compared with TIO at the WTP of 70,000 CNY. IND/GLY should be considered as a good option for stable COPD treatment from Chinese payer's perspective.
OWSA showed that factors such as lung function benefits, treatment exacerbation reducing, and drug costs influenced the results sensitively in this model. The evidence that IND/GLY significantly improves lung function and reduces exacerbation compared with single bronchodilator therapies and ICS therapies is valid in previous randomized controlled trials in China and worldwide. The price of novel drugs and interventions are expected to fall further as the market share is growing and because of government-industry negotiation processing in China. Decreasing prices will make IND/GLY more costeffective. From this we can see that although the results fluctuate sensitively under some scenarios, we can still assume the results of the cost-effectiveness analyses in this model are valid and reliable.

The results of PSA show that IND/GLY is dominantly cost-effective compared with SAL/FLU based on LANTERN trial data. Considering that IND/GLY performed better than SAL/FLU in the LANTERN trial while IND/GLY is at a vlower price than SAL/FLU in the Chinese medical service market, this conclusion is obvious. The results of PSA show that IND/GLY has more than $50 \%$ probability to be cost-effective than TIO at the WTP of $70,000 \mathrm{CNY}$ (one-time GDP per capita in China) as COPD is a chronic disease. WHO recommend to

\section{IND/GLY vs Tiotropium (SPARK)}

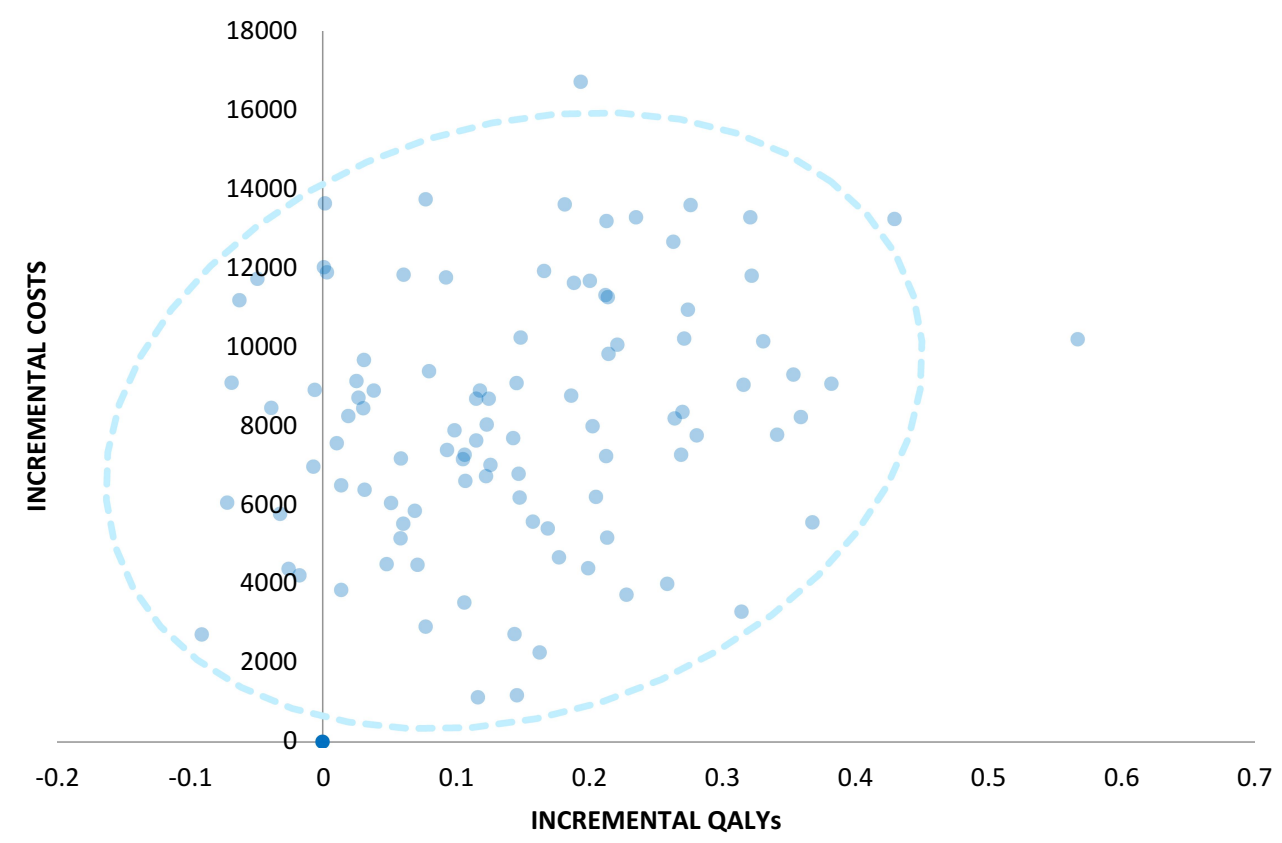

Figure 3 Probability sensitivity analysis of IND/GLY vs tiotropium. We showed the results of probability sensitivity analysis (PSA) of IND/GLY vs tiotropium in this scatter plot. Each scatter represents an iteration in this model with different incremental costs and QALYs in PSA. As IND/GLY can bring longer QALYs but cost more. We need to figure out the probability of scatters to be cost-effective under different threshold. 


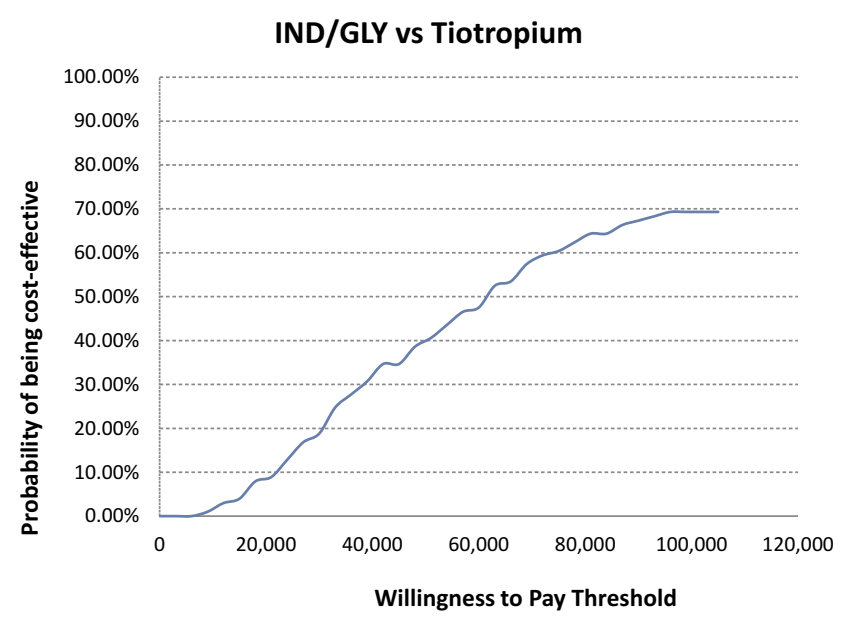

Figure 4 The probability of being cost-effective in PSA of IND/GLY vs tiotropium. This figure showed the probability of iterations in PSA being accepted as costeffective under different threshold. In our study, we selected 70,000 CNY as willingness-to-pay threshold as we mentioned fully in Method section. We can see the probability of cost-effectiveness of IND/GLY comparing with tiotropium is $50-60 \%$.

use 1-3 times of GDP per capita as WTP in cost-utility analysis. $^{22}$ If we select a higher WTP, the conclusion of cost-effectiveness of IND/GLY comparing with TIO will be more significant and valid.

In this study, we used a patient-level simulation model to evaluate cost-effectiveness of IND/GLY and comparators. This model can generate and simulate patients randomly from the mean and distribution of each parameter. Compared with the traditional cohort-level Markov model, the patient-level simulation model is more flexible and accurate in simulating patients' health status. ${ }^{23}$ As current cost-effectiveness analyses studies of IND/GLY in USA and Europe are based on the cohort-level Markov model, ${ }^{17,18}$ our model-based analysis has more advantages in presenting the health status of COPD patients.

Previous studies have proved the cost-effectiveness of IND/GLY over the comparative treatment in the US and Europe. $^{17,18}$ But there are few studies about costeffectiveness analyses for IND/GLY from the Chinese payer's perspective. Because China's economic development level, medical service prices, and ethnic composition are completely different from those of Western countries, the cost-effectiveness needs to be re-evaluated. Our study evaluates the cost-effectiveness of IND/GLY using a model with input sourced from clinical trials and Chinaspecific cost data. Our results are valid in the Chinese medical system setting. Since the cost of IND/GLY is lower than SAL/FLU in China, IND/GLY costs less and has better efficacy than SAL/FLU, which is different with previous studies. As the more cost-effective IND/GLY gradually gains more market share for severe and highrisk COPD in China, the price of SAL/FLU may fall in the future. The cost-effectiveness analyses of IND/GLY comparing with $\mathrm{SAL} / \mathrm{FLU}$ will require re-evaluation at that time.

\section{Limitations}

First, as there is lack of real-world data with long time period of cost and effectiveness of COPD patients with different treatment in China. We did a model-based analysis to simulate real-world situation under Chinese medical system settings. We used a patient-level simulation model as it is more flexible in simulating patients' health status. However, there is still a gap between model simulation and real-world situations. The time duration of clinical trials of COPD treatment is limited. There might be some errors when limited time period trials expanded into model-based whole life simulations. Dual inhaled drugs might be more effective than others in a limited period of time, but there is lack of data on effectiveness beyond the time period of trials. Besides, we may not consider some scenarios as discontinuation of medication, medication for comorbidities, treatment method switches and incorrect inhalations in the model. Considering these dilemmas, there is an urgent need for real-world registry studies to evaluate the effectiveness and cost-effectiveness for COPD patients in China.

Second, costs inputs are derived from a survey over several hospitals. Physicians across China estimate the cost of COPD patients in China from their clinical experience. The results can represent the situation in China in some way, but this may be less reliable than individuallevel data, which is hard to get.

Third, the virtual patients in this model were generated from the characteristics of participants of clinical trials. The distribution of parameters may be different from data from epidemiological investigation for COPD in China. Because the effect of clinical trials is limited to subjects of trials with specific demographic characteristics and specific disease severity, the treatment effect for patients with other characteristics and severity is not a direct reference, so it is hard to simulate and evaluate the treatment effectiveness of real patient groups based on data from clinical trials.

Nowadays, triple bronchodilators therapy was proved to effect better than dual bronchodilators therapy for severe COPD treatment. ${ }^{24,25}$ When these new products come into China, we need to assess their value to guide 
policymaking. There is lack of subgroup analyses of costeffectiveness of IND/GLY. It may perform differently in patients with varying age, sex or disease severity. Besides, as the development of big data, real-world data from health information systems, patient reported outcomes and digital health technology outcomes are more available than before. Considering the limitations of a model-based analyses, future research should focus on comparing the real-world outcomes and the cost-effectiveness of dual bronchodilators in a real-world setting. ${ }^{26}$

\section{Conclusion}

This study found that dual bronchodilator IND/GLY has cost-effectiveness as stable COPD treatment comparing with SAL/FLU and tiotropium under Chinese health care system setting. IND/GLY is acceptable for stable COPD patients in China from Chinese payers' perspective.

\section{Disclosure}

The authors report no conflicts of interest in this work.

\section{References}

1. Wang C, Xu J, Yang L, et al. Prevalence and risk factors of chronic obstructive pulmonary disease in China (the China Pulmonary Health [CPH] study): a national cross-sectional study. The Lancet. 2018;391 (10131):1706-1717. doi:10.1016/S0140-6736(18)30841-9

2. Zhu B, Wang Y, Ming J, Chen W, Zhang L. Disease burden of COPD in China: a systematic review. Int J Chron Obstruct Pulmon Dis. 2018;13:1353. doi:10.2147/COPD.S161555

3. Fang X, Wang X, Bai C. COPD in China: the burden and importance of proper management. Chest. 2011;139(4):920-929. doi:10.1378/ chest.10-1393

4. Xu G, Zhang Z, Lv Q, et al. NSFC health research funding and burden of disease in China. PLoS One. 2014;9(11):e111458. doi:10.1371/journal.pone.0111458

5. Wu M, Zhao Q, Chen Y, Fu C, Xu B. Quality of life and its association with direct medical costs for COPD in urban China. Health Qual Life Outcomes. 2015;13(1):57. doi:10.1186/s12955015-0241-5

6. Singh D, Agusti A, Anzueto A, et al. Global strategy for the diagnosis, management, and prevention of chronic obstructive lung disease: the GOLD science committee report 2019. Eur Respir J. 2019;53(5):5. doi:10.1183/13993003.00164-2019

7. Tashkin DP, Celli B, Senn S, et al. A 4-year trial of tiotropium in chronic obstructive pulmonary disease. $N$ Eng J Med. 2008;359 (15):1543-1554. doi:10.1056/NEJMoa0805800

8. Vogelmeier C, Hederer B, Glaab T, et al. Tiotropium versus salmeterol for the prevention of exacerbations of COPD. $N$ Eng $J$ Med. 2011;364(12):1093-1103. doi:10.1056/NEJMoa1008378

9. Wedzicha JA, Calverley PM, Seemungal TA, Hagan G, Ansari Z, Stockley RA. The prevention of chronic obstructive pulmonary disease exacerbations by salmeterol/fluticasone propionate or tiotropium bromide. Am J Respir Crit Care Med. 2008;177(1):19-26. doi:10.1164/rccm.200707-973OC

10. Calverley PM, Anderson JA, Celli B, et al. Salmeterol and fluticasone propionate and survival in chronic obstructive pulmonary disease. N Eng J Med. 2007;356(8):775-789. doi:10.1056/NEJMoa063070
11. Kardos P, Wencker M, Glaab T, Vogelmeier C. Impact of salmeterol/ fluticasone propionate versus salmeterol on exacerbations in severe chronic obstructive pulmonary disease. Am J Respir Crit Care Med. 2007;175(2):144-149. doi:10.1164/rccm.200602-244OC

12. Anzueto A, Miravitlles M. The role of fixed-dose dual bronchodilator therapy in treating COPD. Am J Med. 2018;131(6):608-622. doi:10.1016/j.amjmed.2017.12.018

13. Wedzicha JA, Decramer M, Ficker JH, et al. Analysis of chronic obstructive pulmonary disease exacerbations with the dual bronchodilator QVA149 compared with glycopyrronium and tiotropium (SPARK): a randomised, double-blind, parallel-group study. Lancet Respir Med. 2013;1(3):199-209. doi:10.1016/S2213-2600(13)70052-3

14. Zhong N, Wang C, Zhou X, et al. LANTERN: a randomized study of QVA149 versus salmeterol/fluticasone combination in patients with COPD. Int J Chron Obstruct Pulmon Dis. 2015;10(1):1015-1026. doi:10.2147/COPD.S84436

15. Bateman ED, Ferguson GT, Barnes N, et al. Dual bronchodilation with QVA149 versus single bronchodilator therapy: the SHINE study. Eur Respir J. 2013;42(6):1484-1494. doi:10.1183/ 09031936.00200212

16. Anzueto AR, Kostikas K, Shen S, et al. Indacaterol/glycopyrronium (IND/GLY) reduces the risk of clinically important deterioration (CID) versus salmeterol/fluticasone (SFC): the FLAME study. Eur Respir Soc. 2017.

17. Rajagopalan K, Bloudek L, Marvel J, Dembek C, Kavati A. Costeffectiveness of twice-daily indacaterol/glycopyrrolate inhalation powder for the treatment of moderate to severe COPD in the US. Int J Chron Obstruct Pulmon Dis. 2018;13:3867. doi:10.2147/COPD. S177097

18. Maleki-Yazdi MR, Molimard M, Keininger DL, et al. Cost effectiveness of the Long-Acting $\beta$ 2-Adrenergic Agonist (LABA)/long-acting muscarinic antagonist dual bronchodilator indacaterol/glycopyrronium versus the LABA/inhaled corticosteroid combination salmeterol/fluticasone in patients with chronic obstructive pulmonary disease: analyses conducted for Canada, France, Italy, and Portugal. Appl Health Econ Health Policy. 2016;14(5):579-594. doi:10.1007/s40258-016-0256-z

19. Organization WH. Healthy China: Deepening Health Reform in China: Building High-Quality and Value-Based Service Delivery. World Bank Publications; 2019.

20. Ip MS-M, Wai-san KF, Lau AC-W, et al. Updated spirometric reference values for adult Chinese in Hong Kong and implications on clinical utilization. Chest. 2006;129(2):384-392. doi:10.1378/chest.129.2.384

21. Rutten-van Mölken MP, Oostenbrink JB, Tashkin DP, Burkhart D, Monz BU. Does quality of life of COPD patients as measured by the generic EuroQol five-dimension questionnaire differentiate between COPD severity stages? Chest. 2006;130(4):1117-1128. doi:10.1378/ chest.130.4.1117

22. Robinson LA, Hammitt JK, Chang AY, Resch S. Understanding and improving the one and three times GDP per capita cost-effectiveness thresholds. Health Policy Plan. 2017;32(1):141-145. doi:10.1093/ heapol/czw096

23. Davis S, Stevenson M, Tappenden P, Wailoo A NICE DSU technical support document 15: cost-effectiveness modelling using patient-level simulation [internet]; 2014.

24. Papi A, Vestbo J, Fabbri L, et al. Extrafine inhaled triple therapy versus dual bronchodilator therapy in chronic obstructive pulmonary disease (TRIBUTE): a double-blind, parallel group, randomised controlled trial. The Lancet. 2018;391(10125):1076-1084. doi:10.1016/ S0140-6736(18)30206-X

25. Cazzola M, Rogliani P, Calzetta L, et al. Triple therapy versus single and dual long-acting bronchodilator therapy in COPD: a systematic review and meta-analysis. Eur Respir J. 2018;52(6):1801586. doi:10.1183/13993003.01586-2018

26. Sherman RE, Anderson SA, Dal Pan GJ, et al. Real-world evidence - what is it and what can it tell us. $N$ Engl J Med. 2016;375 (23):2293-2297. doi:10.1056/NEJMsb1609216 


\section{Publish your work in this journal}

The International Journal of COPD is an international, peer-reviewed journal of therapeutics and pharmacology focusing on concise rapid reporting of clinical studies and reviews in COPD. Special focus is given to the pathophysiological processes underlying the disease, intervention programs, patient focused education, and self management protocols. This journal is indexed on PubMed Central, MedLine and CAS. The manuscript management system is completely online and includes a very quick and fair peer-review system, which is all easy to use. Visit http://www.dovepress.com/testimonials.php to read real quotes from published authors. 